\title{
Effects of Cnidium monnieri (L.) Cuss. fruit extract on sexual behaviors in male rats
}

\author{
Thuy Duong Dau*, Minh Ha Le², Tran Thi Giang Huong Nguyen'1, Thi Ngoc Mai Dang ${ }^{3}$, Thi Nguyet Que Do ${ }^{4}$ and \\ Quynh Trang Tran'
}

\begin{abstract}
Background: Cnidium monnieri (L.) Cuss. has been used for a long time according to the traditional experiences and documentation in order to treat male sexual disorders. Osthole is one of the main bioactive components in Cnidium monnieri (L.) Cuss. In this study, we aimed to evaluate the effect of Cnidium monnieri (L.) Cuss. fruit extract of which osthole content was 35\% (determined by HPLC method) on sexual behaviors in adult male rats.
\end{abstract}

Methods: Cnidium monnieri (L.) Cuss. fruit extract was administered orally at the dose of $150 \mathrm{mg} / \mathrm{kg}$ b.w and after 30 min, male rats were coupled with receptive adult female rats to evaluate sexual behaviors, including mounting and intromission.

Results: Cnidium monnieri (L.) Cuss. fruit extract at the dose of $150 \mathrm{mg} / \mathrm{kg}$ b.w increased the percentage of rats that mounted and intromitted; increased the intromission frequency and decreased mounting latency and intromission latency.

Conclusion: Cnidium monnieri (L.) Cuss. fruit extract increased the ability to mount and intromit in rats, and scientifically support the traditional use of Cnidium monnieri (L.) Cuss. in treatment of male sexual disorders.

Keywords: Cnidium monnieri, Osthole, Male sexual disorders, Rats, Sexual behaviors

\section{Background}

Male sexual disorders including disorders of desire, erectile dysfunction, disorders of ejaculation ... are common among men of all ages, ethnicities and cultures [1]. It has been estimated that more than 152 million men worldwide experienced erectile dysfunction in 1995, and this number will rise by 170 million, to approximately 322 million by the year 2025 [2].

With the significant advances in the field of physiology and pathophysiology, scientists have gained a deep understanding of the etiology, the pathogenesis of male sexual disorders. Since then, the researchers have also made significant strides, finding diagnostic methods and treatments that are suitable for each patient and the cause [1]. In recent years, keeping pace with the trend in

\footnotetext{
* Correspondence: dauthuyduong@hmu.edu.vn

'Department of Pharmacology, Hanoi Medical University, No.1 Ton That

Tung, Dong Da, Ha Noi, Viet Nam

Full list of author information is available at the end of the article
}

the world, Vietnam has applied modern medical treatments in improving reproductive health, and in diagnosing and treating male sexual disorders in particular.

Moreover, like many countries in the world, a common research trend is to discover medicines originating from herbs to treat these disorders. According to traditional experiences and documentation, there are many herbs widely used to treat male sexual disorders. Among them was the Cnidium monnieri (L.) Cuss. According to Do Tat Loi, Cnidium monnieri (L.) Cuss. has the effect of enhancing the sexual function and sexual "power" in men [3]. The main bioactive compound in the Cnidium monnieri (L.) Cuss. fruit is osthole. Osthole was demonstrated to increase smooth muscle relaxation by releasing nitric oxide from endothelium or inhibiting phosphodiesterase [4]. Osthole has also been proved to raise serum testosterone levels and expression of androgens in reproductive decline rat's testes [5]. However, there are few studies of osthole effects on male's sexual 
behaviors. We used alcohol to extract the fruits of Cnidium monnieri (L.) Cuss. The solvent was then removed by rotary evaporation to collect the residue extract. Then the osthole content of $35 \%$ in the extract was determined by using HPLC method. From here on, this extract was referred OS35 in this article. This study was conducted to investigate the effect OS35 on sexual behaviors in adult male rats according to Agmo's method [6].

One of the commonly used experimental models for assessing the effects of herbal medicine on sexual activity is sexual behavior test. Rats are the most common subjects of laboratory research including studies of sexual behavior test and other pharmacological activity. Studies of sexual behavior not only help detect herbal medicine that enhance sexual function, but also orient its mechanism of action.

\section{Methods}

\section{Materials}

The fresh fruits of Cnidium monnieri (L.) Cuss. were harvested in Vietnam, 2017. The fruits were washed, dried and ground into powder. Dried powder of Cnidium monnieri (L.) Cuss. fruits were extracted with alcohol for three times under reflux for $4-5 \mathrm{~h}$. The extract was concentrated to dryness under reduced pressure. The osthole content of the extract determined by HPLC was $35 \%$. Other significant constituent in the extract is imperatorin which occupied of about $9.5 \%$.

Clinical dose of this extract of Cnidium monnieri (L.) Cuss. is about $1500 \mathrm{~g} / 60 \mathrm{~kg}$ person. Rat equivalent dose calculated based on body surface area was $150 \mathrm{mg} / \mathrm{kg}$. The oral administration route in rats was similar to the intended clinical route.

\section{Animals}

Adult Wistar male and female rats approximately 180$220 \mathrm{~g}$ body weight and 8-10 weeks were used. All rats were healthy and drug or test naïve. Rats were housed in pairs in plastic cages at a temperature-controlled room. Rats were provided food and water ad libitum. Experiments were performed in accordance with Vietnamese ethical laws and European Communities Council Directives of November 24, 1986 (86/609/EEC) guidelines for the care and use of laboratory animals.

\section{Procedures}

\section{Habitation to a reversed light-dark cycle}

Male rats usually copulate during the dark phase. This study was carried out in the morning every day. All male and female rats were habituated to a reversed $12 \mathrm{~h}$ light/ $12 \mathrm{~h}$ dark cycle 3 weeks before training sessions (Fig. 1). The light was turned off at $8.00 \mathrm{am}$ and turned on at $8.00 \mathrm{pm}$. During the dark phase, a dim white light was left to handle the animals.

\section{Preparation of sexually receptive females}

Since heterosexual behavior requires individuals of different sex, it is also necessary to prepare sexually receptive females [6, 7]. Female rats were induced sexual reception artificially by ovariectomy under anesthetized 14 days before the first mating (postoperative care was carried out). $52 \mathrm{~h}$ before coupling, each female rat was injected subcutaneously 20 micrograms estradiol benzoate and $1000 \mathrm{mcg}$ progesterone $4 \mathrm{~h}$ later $(48 \mathrm{~h}$ before coupling).

\section{Training sessions}

Each male rats experienced three training sessions (Fig. 1). Male rats were placed in rectangular observatory cage individually. One receptive female rat was introduced to each male rate $5 \mathrm{~min}$ later and male rat's sexual behavior were observed. The following male behaviors were registered: mounting, intromission and ejaculation. The following parameters were evaluated (Fig. 2): ML (mounting latency): time from the introduction of

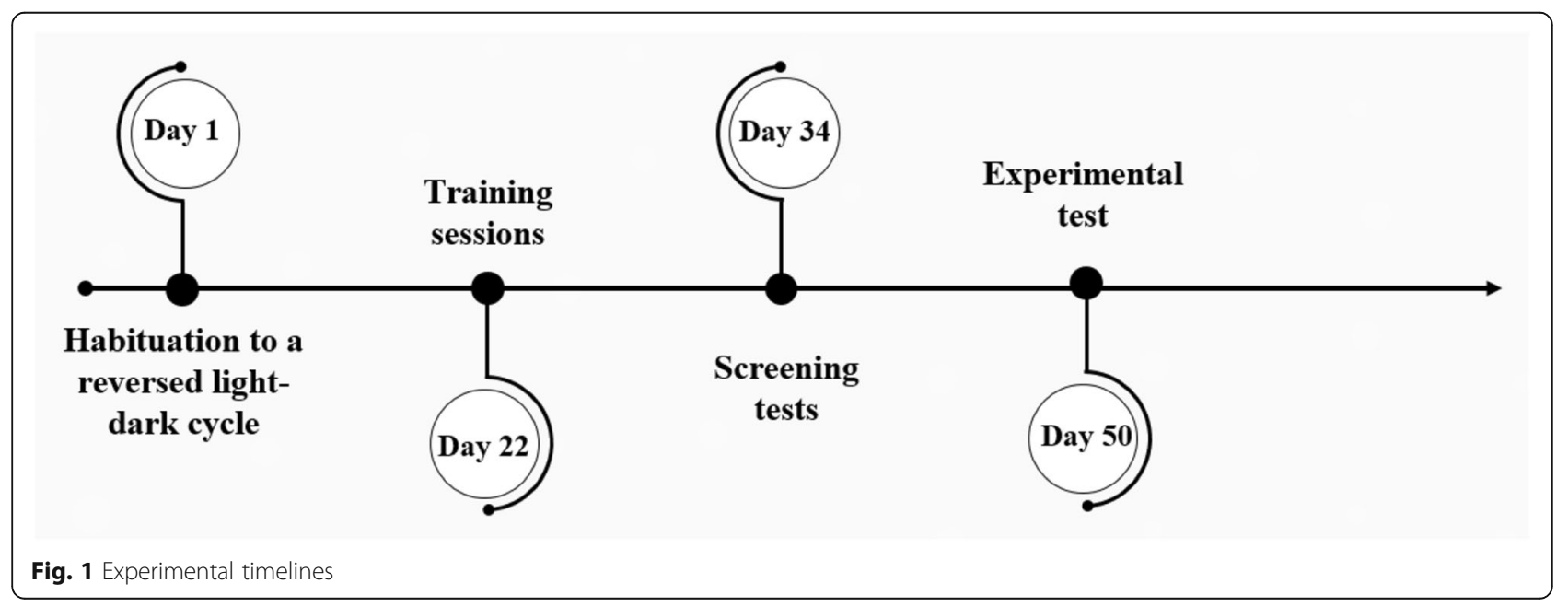


female until the first mount, IF (intromission frequency), IL (intromission latency): time from introduction of female until the first intromission, EL (ejaculation latency): time from the first intromission until ejaculation, PEI (post ejaculation interval): time from ejaculation until the next intromission. The test ended when PEI was recorded or ML, IL > $30 \mathrm{~min}, \mathrm{EL}>30 \mathrm{~min}$, $\mathrm{PEI}>30 \mathrm{~min}$.

Rat coupling was recorded on video and the researchers watched video later to calculate the parameters.

\section{Screening tests}

After training sessions, four screening tests (similar to training test) were conducted to select rats for experimental test (Fig. 1). Rats did not complete a screening test when $\mathrm{ML}>30 \mathrm{~min}, \mathrm{IL}>30 \mathrm{~min}, \mathrm{EL}>30 \mathrm{~min}$ or PEI $>30 \mathrm{~min}$. In this study, those did not complete at least one screening test were considered sexually inactive and were taken into experimental test.

\section{Experimental test}

Twenty-four selected rats from screening tests were randomly divided into 3 groups and were administered tested extract or control drug orally: Group 1: distilled water at $1 \mathrm{ml} / \mathrm{kg}$ b.w/day; Group 2: sildenafil $10 \mathrm{mg} / \mathrm{kg}$ b.w $30 \mathrm{~min}$ before coupling; Group 3: OS35 $150 \mathrm{mg} / \mathrm{kg}$ b.w $30 \mathrm{~min}$ before coupling. Experimental test was similar to screening tests.

Experimental outcomes The experimental outcomes included of percentage of male rats mounted, intromitted; ML, IL and IF.

\section{Statistical analysis}

Data were represented in form of mean \pm standard deviation. All rats in experimental groups were analysed for significance of differences between treatment groups and control groups by Stata 3.0 software. The difference between two groups is judged to be statistically significant when $p \leq 0.05$.

\section{Results}

\section{General animal health status}

Prior to the experiment, all male and female rats were healthy and drug or test naïve. Clinical observations were made every day. No mortality was recorded during the study. No rat showed any clinical symptoms of toxicity or any other adverse events. The body weight was recorded every week and no significant differences of the body weight were found between treated rats and untreated rats prior to or during the tests.

\section{Effect of OS35 on mounting}

According to Fig. 3 and Table 2, sildenafil and OS35 increased the rate of mounting rats and shortened ML statistically compared to untreated control group $(p<0.05)$.

\section{Effect of OS35 on intromission}

Our results showed that sildenafil increased the rate of intromitting rats (Fig. 3); increased IF compared with untreated rats $(\mathrm{p}<0.05)$ (Table 1) and decreased IL compared with untreated rats significantly $(\mathrm{p}<0.05)$ (Table 2).

\section{Discussion}

Adult male rats are used because they only perform sexual intercourse when there is normality of the hypothalamus-pituitary-gonads axis. Moreover, most studies of male rat sexual behavior use sexually experienced rats [6]. Therefore, it is necessary to allow the animals to acquire limited but sufficient sexual experience. In this study, rats had to undergo three training sessions (to become familiar with the behavioral test and the initiation of sexual reflexes) before screening tests.

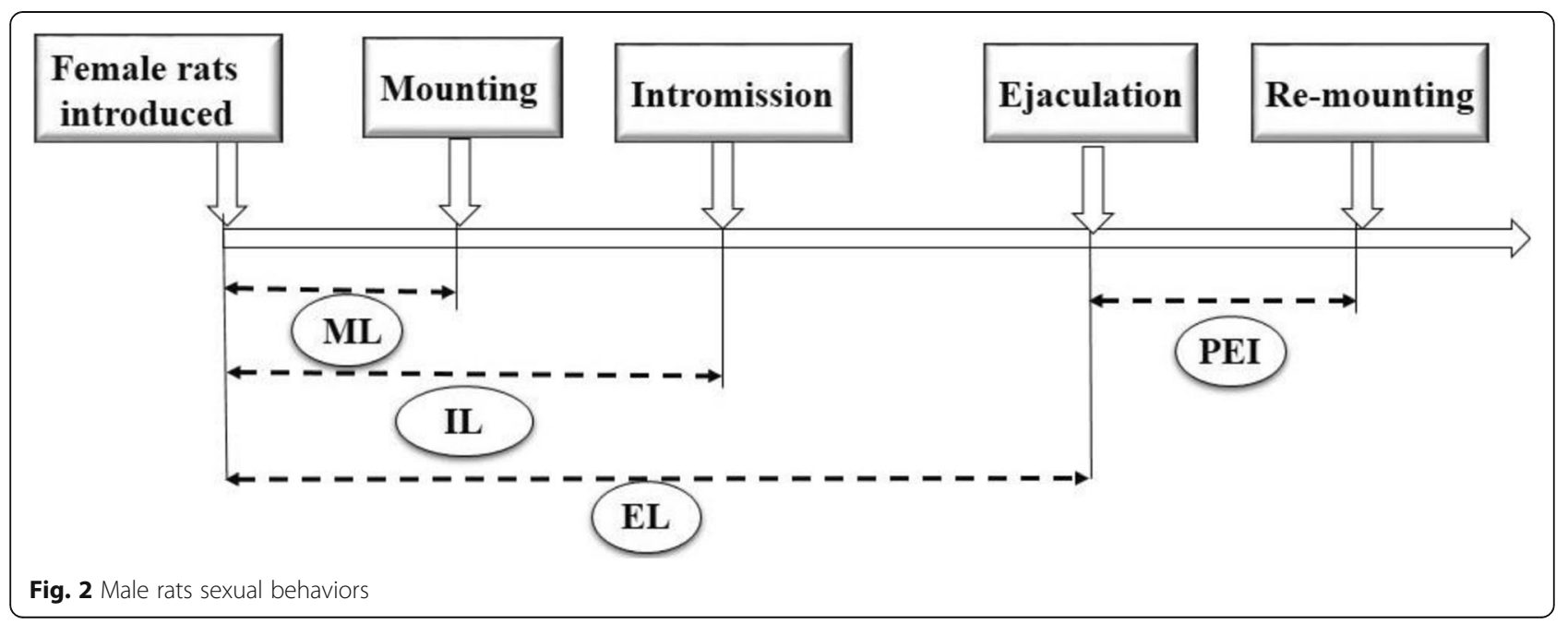




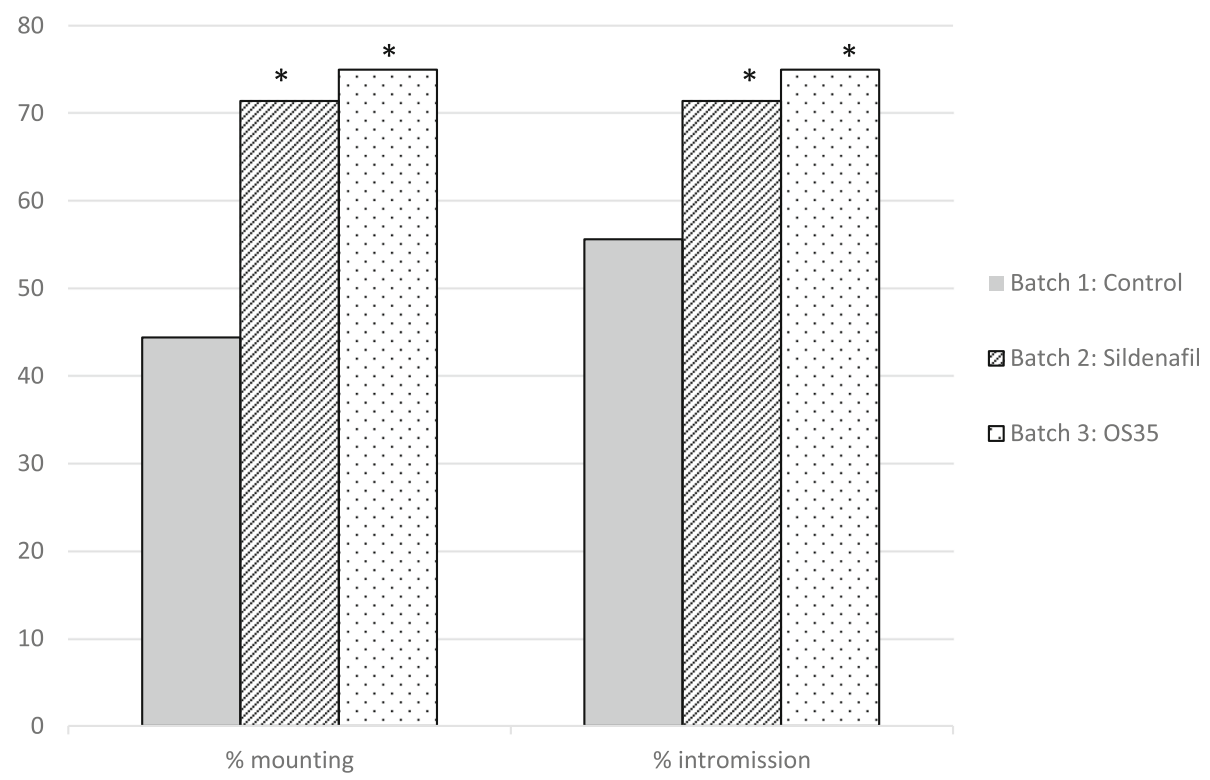

Fig. 3 Effects of OS35 on the percentage of male rats mounted, intromitted

Rely on changes in evaluated parameters, Agmo's method does not require complicated machinery and can be carried out easily in developing country. Sildenafil is chosen as active control drug since this substance is a potent and selective phosphodiesterase type $\mathrm{V}$ inhibitor and is indicated to treat erectile dysfunction in adult men, who are unable to achieve or maintain erection sufficient for satisfactory sexual performance.

The male rat's sexual behaviors is activated by stimuli emitted by the receptive female, including 3 actions: mounting, intromission and ejaculation, and male rats will remount after an interval from ejaculation $[6,7]$.

\section{Sexual desire}

Mounting reflects sexual activation or sexual desire, associated with libido in humans. ML is an important parameter of sexual desire. Our results indicated that sildenafil and OS35 decreased ML in rats. Therefore, sildenafil and OS35 had the potential to increase sexual desire.

How did sildenafil and OS35 change sexual desire? Some scientific evidence proves the role of central dopaminergic system in regulating sexual behavior, including sexual desire in humans and animals. Similar results were obtained from other authors' research including [8-11] etc. To explain this effect of sildenafil, scientists have theorized the effect of sildenafil on the central nervous system, specifically on the central dopaminergic system [12, 13]. In 2013, Kyratsas C. clarified this effect by administering sildenafil to male rats, then quantifying dopamine and its metabolites by HPLC in the medial preoptic area (mPOA) and nucleus accumbens (NAcc). The results showed that sildenafil increased the activity of dopaminergic system in the medial preoptic area (mPOA) and nucleus accumbens (NAcc). The authors suggested that sildenafil might have an effect on the central nervous pathway involved in sexual arousal [13].

Similar results were found in the OS35 group, OS35 at the dose of $150 \mathrm{mg} / \mathrm{kg} \mathrm{b.w}$ increased the rate of mounting rats and shortened ML. Osthole has been demonstrated to increase nitric oxide (NO) release from the cavernous endothelium [4], so it has not been excluded that osthole also has the effect of increasing NO release from vascular smooth muscle. In addition, [14] found that the osthole and imperatorin increased the release of glutamate from neurons. Then [15] suggested that the effect of osthole glutamate release was related to
Table 1 Effects of OS35 on IF

\begin{tabular}{ll}
\hline Group & $\begin{array}{l}\text { Median (min - max) } \\
\text { IF }\end{array}$ \\
\hline Group 1: control & $9(0-29)$ \\
Group 2: sildenafil & $26(22-30)^{*}$ \\
Group 3: OS35 & $24(1-36)^{*}$ \\
\hline
\end{tabular}

"Statistically significant compared with control group (group 1)
Table 2 Effects of OS35 on ML, IL

\begin{tabular}{lll}
\hline Groups & ML (seconds & IL (seconds) \\
\hline Group 1: control & $1019.40 \pm 308.60$ & $844.11 \pm 302.62$ \\
Group 2: sildenafil & $32.20 \pm 5.93$ & $391.16 \pm 282.71^{*}$ \\
Group 3: OS35 & $18.00 \pm 10.27$ & $52.50 \pm 21.38^{*}$ \\
\hline
\end{tabular}

"Statistically significant compared with control group (group 1) 
activating GMP / PKG-dependent pathways. So, how does NO and glutamate relate to sexual desire? The main factors that regulate dopamine release in the anterior preoptic area are sensory stimuli and testosterone. In addition, the results of some studies showed that NO and glutamate also have this effect [16]. As such, this may be a hypothesis of the mechanism of action of OS35 increasing sexual desire in male rats. However, more further studies needed to demonstrate this hypothesis.

\section{Sexual potency}

Vaginal intromission requires an erection. Erection requires a combination of vasodilatation, hormones, and neurotransmitters. Thus, intromission activity is characterized by sexual potency or sexual intercourse. IF index not only shows erectile function but also demonstrates the ability to maintain erections in order to conduct intercourse and premise ejaculation.

In this study, sildenafil increased IF in rats. This result is perfectly consistent with the mechanism of action of sildenafil PDEV inhibition, which increases the amount of cGMP that causes vasodilatation, increases intracavernosum pressure, increases erectile function $[17,18]$. Increased intromission, enhanced sexual intercourse and improved erectile function of sildenafil were also reported in many studies using sildenafil as a positive control [8-11].

In rats given OS35 at the dose of $150 \mathrm{mg} / \mathrm{kg}$ b.w, the percentage of intromitting rats and IF were statistically significantly higher than control rats. James Chen et al. (2000) assessed the effect of osthole on the relaxation of rabbit corpus cavernosum tissue in vitro. Results showed that osthole had the effect of relaxing the smooth muscle of the corpus cavernous [4]. Tetrodotoxin, a neurotransmitter, did not alter osthole's that relaxation, so the effect of osthole was not related to nerves but through vascular endothelial mechanisms. The main mechanism of smooth muscle relaxing action of osthole was increasing NO release from endothelial blood vessels. To confirm this, the researchers removed the cavernous vascular endothelium, which showed a significant reduction in osthole's ability to induce smooth muscle relaxation. Similar results were observed in the presence of L-NAME (N-omega-Nitro-L-arginine methyl ester hydrochloride, a nitric oxide synthase inhibitor) or ODQ (guanyl cyclase inhibitor). Thus, endothelial function is closely related to the effect of osthole [4].

However, the scientists also found that the removal of the cavernous endothelium and the presence of LNAME or ODQ, although significantly reducing osthole's smooth muscle relaxation, but this action did not completely disappear but still partially worked. In addition, in the presence of forskolin (the activator of adenyl cyclase), osthole's smooth muscle relaxation effects were increased compared to that without forskolin [4]. This showed that the mechanism of osthole was not only related the NO pathway but also related to the direct action on the cavernous smooth muscle.

Moreover, when evaluating the effect of OS35 on intromission, OS35 also reduced IL. IL index reflects both sexual intercourse and sexual libido. Thus, OS35 not only increases sexual intercourse, but can also be able to increase libido (as mentioned above). Results from this study showed that Cnidium monnieri (L.) Cuss. fruit extract had potential to increase erectile function and sexual desire as well. The effect of OS35 on sexual behaviors is probably caused by a synergistic effect of the two coumarin derivatives, osthole and imperatorin. However, we need further studies to demonstrate the effectiveness as well as the mechanism of this extract.

\section{Conclusions}

Our study demonstrated that Cnidium monnieri (L.) Cuss. fruit extract (containing $35 \%$ of osthole) at the dose of $150 \mathrm{mg} / \mathrm{kg}$ b.w had good effect on male sexual behaviors in rat model by increasing percentage of mounting and intromitting rats, increasing intromission frequency and decreasing mounting latency and intromission latency. It might explain the empirical application of Cnidium monnieri (L.) Cuss. in treatment of male sexual disorders.

\section{Abbreviations \\ HPLC: High Performance Liquid Chromatography; ML: Mounting latency; IF: Intromission frequency; IL: Intromission latency; EL: Ejaculation latency; PEI: Post ejaculation interval; NO: Nitric oxide; PDEV: Phosphodiesterase type V; cGMP: Cyclic guanosine monophosphate; GMP/PKG: cGMP-dependent protein kinase; L-NAME: N-omega-Nitro-L-arginine methyl ester hydrochloride; ODQ: Guanyl cyclase inhibitor}

\section{Acknowledgements}

We are thankful to Vietnam Academy of Science and Technology (VAST), Hanoi Medical University and Hanoi University of Pharmacy for supporting us to carry out this study.

\section{Authors' contributions}

DDT, HLM, QDTN, HNTTG did the literature review and designed the experiments. All the authors performed the experiments. DDT, QDTN, MDTN analysed and interpreted the data. DDT prepared the manuscript. HNTTG, HLM, MDTN checked and edited the manuscript. All authors revised and approved the submitted manuscript.

\section{Funding}

Not applicable.

\section{Availability of data and materials}

The dataset used during the current study are available from the corresponding author on reasonable request.

Ethics approval and consent to participate

Experiments were performed in accordance with Vietnamese ethical laws and European Communities Council Directives of November 24, 1986 (86/ $609 / \mathrm{EEC}$ ) guidelines for the care and use of laboratory animals. 


\section{Consent for publication}

Not applicable.

\section{Competing interests}

The authors declare that they have no competing interests.

\section{Author details}

'Department of Pharmacology, Hanoi Medical University, No.1 Ton That Tung, Dong Da, Ha Noi, Viet Nam. ${ }^{2}$ Institute of Natural Product Chemistry, Vietnam Academy of Science and Technology, Ha Noi, Viet Nam. ${ }^{3}$ Centre of Clinical Pharmacology, Hanoi Medical University, Ha Noi, Viet Nam. ${ }^{4}$ Department of Pharmacology, Hanoi University of Pharmacy, Ha Noi, Viet Nam.

Received: 29 April 2020 Accepted: 10 November 2020

Published online: 25 November 2020

\section{References}

1. Fouad RK, Vivien KTK, Ronald SS. Male sexual function and its disorders: physiology, pathophysiology, clinical investigation, and treatment. Endocr Rev. 2001;22(3):342-6. https://doi.org/10.1210/edrv.22.3.0430.

2. Ayta I, McKinlay J, Krane R. The likely worldwide increase in erectile dysfunction between 1995 and 2025 and some possible policy consequences. Br J Urol Int. 1999;84:50-7. https://doi.org/10.1046/j.1464410x.1999.00142.x

3. Do TL. Vietnames medicinal plants and herbs. Hanoi: Medical Publishing House; 2004.

4. Chen J, Chiou W-f, Chen C-C, Chen C-F. Effect of the plant-extract osthol on the relaxation of rabbit corpus cavernosum tissue in vitro. J Urol. 2000;163: 1975-6. https://doi.org/10.1097/00005392-200006000-00105

5. Yuan J, Xie J, Li A, Zhou F. Effects of Osthole on androgen level and nitric oxide synthetase activity in castrated rats. Zhong Yao Cai. 2004;27(7):504-3

6. Ågmo A. Protocol male rat sexual behavior. Brain Res Protocol. 1997;1:2037. https://doi.org/10.1016/S1385-299X(96)00036-0.

7. Hull E, Dominguez J. Sexual behavior in male rodents. Horm Behav. 2007; 52(1):45-11. https://doi.org/10.1016/j.yhbeh.2007.03.030.

8. Tajuddin, Shamshad A, Abdul L. An experimental study of sexual function improving effect of Myristica fragrans Houtt. (nutmeg). BMC Complement Altern Med. 2005:5(16). https://doi.org/10.1186/1472-6882-5-16.

9. Senbel A, Mostafa T. Yohimbine enhances the effect of sildenafil on erectile process in rats. Int J Impotance Res. 2008;20:409. https://doi.org/10.1038/sj. ijir.3901630.

10. Fouche G, Anthony J, Olubunmi A. Effect of the aqueous extract of the aerial parts of Monsonia angustifolia E.Mey.Ex.A.Rich., on the sexual behavior of male Wistar rats. BMC Complement Altern Med. 2015;15:353. https://doi. org/10.1186/s12906-015-0880-4

11. Erhabor JO, Idu MD. Aphrodisiac potentials of the ethanol extract of Aloe barbadensis mill. Root in male Wistar rats. BMC Complement Altern Med. 2017;17:360. https://doi.org/10.1186/s12906-017-1866-1.

12. Giuliani D, Ottani A, Ferrari F. Influence of sildenafil on copulatory behavior in sluggish or normal ejaculator male rats: a central dopamine mediated effect? Neuropharmacology. 2002;42(4):562-6. https://doi.org/10.1016/s00283908(01)00195-2.

13. Kyratsas C, Dalla C, Anderzhanova E. Experimental evidence for sildenafil's action in the central nervous system: dopamine and serotonin changes in the medial preoptic area and nucleus accumbens during sexual arousal. J Sex Med. 2013;10(3):719-1. https://doi.org/10.1111/j.1743-6109.2012.03000.x.

14. Wang $S$, Lin T, Lu C, Huang W. Osthole and imperatorin, the active constituents of Cnidium monnieri (L.) Cusson, facilitate glutamate release from rat hippocampal nerve terminals. Neurochem Int. 2008;53(6-8):416-8. https://doi.org/10.1016/j.neuint.2008.09.013.

15. Lin T, Lu C, Huang W, Wang S. Involvement of the CGMP pathway in the osthole-facilitated glutamate release in rat hippocampal nerve endings. Synapse. 2012;66(3):232-8. https://doi.org/10.1002/syn.21505.

16. Juan M, Elaine M. Dopamine, the medial preoptic area, and male sexual behavior. Physiol Behav. 2005;86:356-13. https://doi.org/10.1016/j.physbeh. 2005.08.006

17. Rang H, Dale M. Rang and Dale's Pharmacology. Endinburgh, New York: Elsevier/Churchill Living Stone; 2012. p. 417-32.

18. Goldstein I. Oral sildenafil in the treatment of erectile dysfunction. J Urol. 2002;167(2):1197. https://doi.org/10.1016/S0022-5347(02)80386-X.

\section{Publisher's Note}

Springer Nature remains neutral with regard to jurisdictional claims in published maps and institutional affiliations.

\section{Submit your manuscript to a SpringerOpen ${ }^{\circ}$ journal and benefit from:}

- Convenient online submission

- Rigorous peer review

- Open access: articles freely available online

- High visibility within the field

- Retaining the copyright to your article

Submit your next manuscript at $\boldsymbol{\nabla}$ springeropen.com 